\title{
A single-center experience with 12 consecutive cases of pregnancy among patients with membranous ventricular septal aneurysm
}

Kana Wang ${ }^{1,2}$, Xiaodong Wang ${ }^{1,2}$, Haiyan Yu ${ }^{1,2^{*}}$, Xinghui Liu ${ }^{1,2}$, Aiyun Xing ${ }^{1,2}$ and Yong You ${ }^{1,2}$

\begin{abstract}
Background: Membranous ventricular septal aneurysm (MVSA) is a rare cardiac anomaly that can occur as an isolated entity or being associated with other cardiac malformations. Complications of MVSA include thromboembolism, arrhythmia, rupture, bacterial endocarditis, right ventricular outflow tract obstruction, and atrioventricular valve diseases.The success rate of pregnancy and delivery in patients with MVSA has not been reported in the literature. This study was to assess the clinical implications of this condition from our center's experience.

Methods: This was a retrospective study for consecutive 12 pregnancies in women with MVSA, who delivered at a tertiary care center in west China between May 2008 and March 2015.

Results: All patients with MVSA delivered via caesarian section. One patient with severe pulmonary arterial hypertension expired from pulmonary infection and heart failure after delivery. One patient terminated pregnancy in the second trimester- necessitated by cardiogenic shock. The other mothers had varying degrees of cardiac morbidity, but survived. Ten of thirteen newborns survived. Congenital heart disease and small-for-gestational-age (SGA) of newborn occurred in two cases (one twin and one single gestation). Two of these babies expired.

Conclusions: Maternal and neonatal risk appeared associated with heart functional classifications, pulmonary hypertension and histories of cardiac events such as serious cardiac arrhythmia. Accurate diagnosis and care by a multidisciplinary team is recommended for pregnant woman with MVSA.
\end{abstract}

Keywords: Membranous ventricular septal aneurysm (MVSA), Congenital heart disease, Pregnancy outcome

\section{Background}

Membranous ventricular septal aneurysm (MVSA) is an uncommon anomaly of the membranous portion of the cardiac septum. Aneurysmal bulging begins in a weak or incomplete cardiac septum that is unable to overcome the differential pressure between the left and right ventricles. MVSA occurs as an isolated abnormality or being associated with other cardiac malformations. MVSA is a well-recognized risk factor for outflow tract obstruction and embolisms, and is related (either

\footnotetext{
*Correspondence: fanjy422@163.com

'Department of Obstetrics and Gynecology, West China Second University

Hospital, Sichuan University, Chengdu, China

${ }^{2}$ Key Laboratory of Birth Defects and Related Diseases of Women and

Children, (Sichuan University), Ministry of Education, Chengdu, China
}

directly or secondary to) to rupture, bacterial endocarditis, and arrhythmia. However, most patients with this cardiac anomaly were asymptomatic, and the aneurysms were discovered incidentally on echocardiography. Without diagnosis, unforeseen problems or unexpected deaths may occur in patients who are under a conditions of increased cardiovascular stress, such as pregnancy. The outcome of pregnancy and delivery in patients with MVSA has not been reported in the literature. We present 12 cases of pregnant women with incidentally discovered MVSA and discussed our experience in managing these cases. 


\section{Methods}

This was a retrospective study approved by the Institutional Review Board of West China Second University Hospital. We reviewed the medical records of 591 pregnant women with congenital heart disease (CHD) who were admitted at West China Second University Hospital, a tertiary referral center, between May 2008 and March 2015. Prevalence of CHD in pregnancy was $0.88 \%$ (591/66988). Among 591 pregnant women with CHD, 12 cases of MVSA (2.03\%) were diagnosed.

\section{Patient characteristics}

The age of 12 pregnant women with MVSA ranged from 17 to 34 years old. All patients underwent clinical physical examination, electrocardiography (ECG), and transthoracic echocardiography. The co-morbidities of hypertension, diabetes mellitus, and other pregnancy associated significant conditions were also recorded and analyzed in the current study.

\section{Echocardiography}

All included patients were evaluated by echocardiography. MVSA was diagnosed when the protrusion in the echocardiographic evaluation exceeded $7.5 \mathrm{~mm}$ [1]. The range of the protrusion, the mobility of the aneurismal structure, the size of the defect, left ventricular systolic function and the presence of other heart abnormalities were measured. Color flow Doppler was used to evaluate for the presence of septal shunt and valvular regurgitation, and to assess pulmonary artery pressure gradients. The left atrial/ventricular size or diameter was assessed in the transverse dimension in the parasternal longitudinal view (M-mode) and calculated planimetrically in the apical four-chamber view (2D). If mural thrombus was suspected, further echocardiographic evaluation was performed.

\section{Electrocardiogram}

24-h ambulatory ECGs were performed in patients when they had abnormal electrocardiographic pattern; or in patients with abnormal electrolyte that might increase susceptibility to arrhythmia.

\section{Other examinations}

MRI was used to confirm ischemic stroke. Vascular ultrasound was performed for patient with suspected peripheral embolism. Central venous catheterization (CVT) and/or right heart catheterization was undertaken to monitor central venous pressure (CVP) and pulmonary artery pressure gradients in patients who had signs of pulmonary hypertension.

\section{Results}

\section{Cardiac complications}

Eight cases of MVSA, heart disease was initially diagnosed during pregnancy. Five of these patients presented with chest tightness and shortness of breath at the second trimester. Three asymptomatic cases were found on the basis of systolic murmurs upon ausculation, and confirmed by echocardiography during the second trimester. In the remaining 4 cases (33.3\%), diagnosis was made for the first time during labour. Cardiographic characteristics of the mothers are summarized in Table 1. Nine (75\%) cases had cardiac function NYHA class III or higher. Five cases $(41.7 \%)$ of MVSA existed in isolation, and $7(58.3 \%)$ cases were complicated with ventricular septal defect.

Upon diagnosis, the diameter of MVSA ranged from $8 \mathrm{~mm}$ to $28 \mathrm{~mm}$ (mean \pm SD: $13.54 \pm 4.31$ ) and the protrusion area from $8 \times 8 \mathrm{~mm}$ to $28 \times 14 \mathrm{~mm}$ ). Among them, 6 cases had a diameter greater than $15 \mathrm{~mm}$. The aneurysm remained intact in 4 cases and was ruptured in 2 cases. Left cardiac diameter and left ventricular systolic function were assessed in all patients. Enlarged left heart chamber was found in 5 patients, and limited systolic function ( $\mathrm{EF}=30 \%, \mathrm{FS}=14 \%$ ) was found in 1 patient.

In our study, the most common complications attributable to MVSA were pulmonary arterial hypertension (PAH) (58.3\%), atrioventricular valve diseases (66.7\%) and arrhythmias (58.3\%). No thromboembolism was found in our cases. There were 3 cases (25\%) of mild $\mathrm{PAH}, 3$ cases $(25 \%)$ of moderate $\mathrm{PAH}$ and 1 case $(8.3 \%)$ of severe PAH. Eight patients had tricuspid and/or mitral regurgitation but none of them developed stenosis. According to ECG, one patient had 1st-degree ventricular premature beats (I AVB) and ventricular premature beats (VPB). One patient had atrial premature beats (APB); 3 patients had sinus tachycardia, and 1 patient had incomplete right bundle branch block.

\section{Obstetric complications}

The clinical data of obstetric complications, maternal mortality and fetal/neonatal outcome are shown in Table 2. One case (NYHA class IV with severe pulmonary hypertension) had to terminate pregnancy at 17 weeks because of cardiogenic shock. She was then transferred to the Department of Cardiology for further treatments. Obstetric complications were found in 9 $(81.8 \%)$ patients, including premature labor, premature rupture of membranes (PROM), preeclampsia, breech presentation, gestational diabetes mellitus (GDM), and postpartum hemorrhage resulting from twin pregnancy and placenta praevia. All cases had elective cesarean sections. Eight patients received combined spinalepidural anesthesia, 1 epidural anesthesia, and 2 patients general anesthesia. 
Table 1 Characteristics of echocardiography and electrocardiogram in pregnant woman with MVSA

\begin{tabular}{|c|c|c|c|c|c|c|c|c|c|c|}
\hline No. & $\begin{array}{l}\text { Type of } \\
\text { heart disease }\end{array}$ & $\begin{array}{l}\text { LA/LV } \\
\text { diameter (mm) }\end{array}$ & NYHA-FC & $\begin{array}{l}\text { Range of } \\
\text { protrusion } \\
(\mathrm{mm})\end{array}$ & $\begin{array}{l}\text { Size of } \\
\text { the defect } \\
(\mathrm{mm})\end{array}$ & $\mathrm{PH}^{*}$ & $\begin{array}{l}\text { Valve } \\
\text { condition }\end{array}$ & $\begin{array}{l}\text { Mural } \\
\text { thrombus }\end{array}$ & LVSD $^{*}$ & Arrhythmia \\
\hline 1 & VSA & Normal & $\|$ & $13 \times 13$ & No break & None & $\mathrm{TR}^{*}$ & None & $\begin{array}{l}E F=59 \%, \\
F S=31 \%\end{array}$ & - \\
\hline 2 & $\begin{array}{l}\text { VSA } \\
\text { VSD* }\end{array}$ & Normal & III & $15 \times 11$ & 5 & None & Normal & None & $\begin{array}{l}E F=57 \% \\
F S=30 \%\end{array}$ & - \\
\hline 3 & $\begin{array}{l}\text { VSA } \\
\text { VSD }\end{array}$ & Normal & III & $16 \times 15$ & 11 & Moderate & TR & None & $\begin{array}{l}\mathrm{EF}=71 \%, \\
\mathrm{FS}=41 \%\end{array}$ & Sinus tachycardia \\
\hline 4 & $\begin{array}{l}\text { VSA } \\
\text { VSD }\end{array}$ & $L A=38, L V=52$ & III & $9 \times 8$ & 5 & Mild & $M R^{*}$ & None & $\begin{array}{l}E F=59 \% \\
F S=33 \%\end{array}$ & - \\
\hline 5 & $\begin{array}{l}\text { VSA } \\
\text { VSD } \\
\text { PDA* }\end{array}$ & Normal & III & $17 \times 12$ & 5 & Mild & $M R+T R$ & None & $\begin{array}{l}E F=60 \%, \\
F S=32 \%\end{array}$ & Sinus tachycardia \\
\hline 6 & $\begin{array}{l}\text { VSA } \\
\text { VSD }\end{array}$ & $\mathrm{LA}=35, \mathrm{LV}=58$ & III & $14 \times 11$ & 11 & Moderate & Normal & None & $\begin{array}{l}E F=58 \%, \\
F S=31 \%\end{array}$ & $\begin{array}{l}\text { Sinus rhythm with extreme } \\
\text { right axis deviation }\left(+85^{\circ}\right)\end{array}$ \\
\hline 7 & $\begin{array}{l}\text { VSA } \\
\text { VSD }\end{array}$ & $L A=33, L V=50$ & III & $14 \times 14$ & 7 & Moderate & TR & None & $\begin{array}{l}E F=64 \%, \\
F S=34 \%\end{array}$ & - \\
\hline 8 & VSA & $L A=46, L V=57$ & III-IV & $28 \times 14$ & No break & Mild & $M R+T R$ & None & $\begin{array}{l}\mathrm{EF}=30 \%, \\
\mathrm{FS}=14 \%\end{array}$ & $I^{\circ} A V B, V P B$ \\
\hline 9 & $\begin{array}{l}\text { VSA } \\
\text { VSD }\end{array}$ & $\mathrm{LA}=38, \mathrm{LV}=57$ & IV & $20 \times 15$ & 8 & Severe & TR & None & $\begin{array}{l}E F=59 \% \\
F S=32 \%\end{array}$ & IRBBB \\
\hline 10 & VSA & Normal & $\|$ & $12 \times 12$ & 4 & None & Normal & None & $\begin{array}{l}E F=71 \% \\
F S=40 \%\end{array}$ & Sinus tachycardia \\
\hline 11 & VSA & Normal & III & $16 \times 10$ & No break & None & $M R+T R$ & None & $\begin{array}{l}E F=63 \%, \\
F S=34 \%\end{array}$ & Sinus arrhythmia, APB \\
\hline 12 & VSA & Normal & $|-| \mid$ & $8 \times 8$ & No break & None & Normal & None & $\begin{array}{l}\mathrm{EF}=69 \% \\
\mathrm{FS}=39 \%\end{array}$ & - \\
\hline
\end{tabular}

VSD ventricular septal defect, PDA patent ductus arteriosus, $P F O$ patent foramen ovale, NYHA-FC cardiac function grading (New York Heart Association), $P H$ pulmonary hypertension, $L V S D$ left ventricular systolic function, $L A / L V$ Left atrial/ventricular, $M R$ mitral regurgitation, $T R$ tricuspid regurgitation, $A V B$ atrioventricular block, IRBBB incomplete right bundle branch block, VPB ventricular premature beat, $A P B$ atrial premature beat

\section{Maternal and perinatal outcomes}

In this study, 6 (50\%) woman were primigravid (Table 2). The case fatality rate in the study was $8.3 \%$ with death resulting from pulmonary infection and heart failure. Most neonates, 10 (76.9\%) including a twin pregnancy had birth weights more than $2000 \mathrm{~g}$ but less than $2500 \mathrm{~g}$. Three neonates (38.5\%) weighed more than $2500 \mathrm{~g}$. Neonatal case fatality rate was $15.4 \%$. Congenital heart disease was noted in 2 babies (15.4\%), one with TOF, and the other with PDA and PFO. The rate of small-forgestational-age (SGA) (estimated infant weight after delivery below the 10th percentile for corresponding gestational age) was $15.4 \%$.

Of note, maternal and neonatal mortality occurred only in patient with a MVSA protrusion diameter $>15 \mathrm{~mm}$ and NYHA class III-IV. The maternal cardiac anomaly and the outcome of pregnancies are summarized in Table 3.

\section{Discussion}

Ventricular septal aneurysms are extremely rare clinicopathological entities that include congenital muscular interventricular septal aneurysm (CMISA) and membranous ventricular septal aneurysm (MVSA) $[2,3]$.
Membranous ventricular septal aneurysm was first described as a congenital malformation in 1826 by Laennec [4]. It is thought that the membranous septum remains weak during embryonic development, and forms an aneurysm of the membranous septum-bulging toward the right under the pressure of left ventricle. It has also been proposed that MVSA could be acquired-the consequence of delayed natural closure of a ventricular septal defect after birth [5-7]. Indeed, MVSA is primarily associated with a history of spontaneous closure of a small membranous ventricular septal defect in childhood [2]. Patients with MVSA may incur potential cardiac complications, such as aortic valve prolapse, right ventricular outflow obstruction, tricuspid valve insufficiency, arrhythmia, rupture, thromboembolism, and bacterial endocarditis [8-12]. However, most MVSA patients are asymptomatic, the physical examination and electrocardiogram may fail to identify MVSA in the absence of ventricular arrhythmia or other complications [13]. MVSA may provide an occult cardiac source of cerebral or systemic embolus [14]. Furthermore, congestive heart failure may occur because of adverse effects of MVSA on systolic function [9]. 
Table 2 Obstetrics characteristics of pregnancy complicated with MVSA

\begin{tabular}{|c|c|c|c|c|c|c|c|c|c|c|}
\hline No. & Age & $\mathrm{BMl}$ & $\begin{array}{l}\text { Gravidity and } \\
\text { parity history }\end{array}$ & $\begin{array}{l}\text { Obstetric } \\
\text { complication }\end{array}$ & $\begin{array}{l}\text { Mode and time } \\
\text { of delivery }\end{array}$ & $\begin{array}{l}\text { Anesthesia } \\
\text { methods }\end{array}$ & $\begin{array}{l}\text { Apgar Score } \\
\text { (1-5-10 min) }\end{array}$ & Outcomes & $\begin{array}{l}\mathrm{HOD} \\
\text { (day) }\end{array}$ & $\begin{array}{l}\text { Antibiotics } \\
\text { and day uses }\end{array}$ \\
\hline 1 & 29 & 26.56 & G1P0 & None & $\begin{array}{l}\text { Caesarean } \\
\text { section: } \\
38+2 \text { weeks }\end{array}$ & $\begin{array}{l}\text { Combined } \\
\text { spinal epidural }\end{array}$ & $10-10-10$ & $\begin{array}{l}M \text { - good } \\
N \text { - live, } 3175 \mathrm{~g}\end{array}$ & 6 & Ceftezole (5d) \\
\hline 2 & 17 & 25.08 & G1P0 & $\begin{array}{l}\text { Prematurity, } \\
\text { Preeclampsia } \\
\text { Breech } \\
\text { presentation }\end{array}$ & $\begin{array}{l}\text { Caesarean } \\
\text { section: } \\
31+1 \text { weeks }\end{array}$ & $\begin{array}{l}\text { Epidural } \\
\text { anesthesia }\end{array}$ & $10-10-10$ & $\begin{array}{l}M \text { - good } \\
N \text { - live, } 2050 \mathrm{~g}, \\
\text { with PDA and PFO }\end{array}$ & 9 & Cefoxitin (2.5d) \\
\hline 3 & 32 & 27.04 & G3P1 & GDM & $\begin{array}{l}\text { Caesarean } \\
\text { section: } \\
37 \text { weeks }\end{array}$ & $\begin{array}{l}\text { General } \\
\text { anesthesia }\end{array}$ & $10-10-10$ & $\begin{array}{l}M \text { - good } \\
\mathrm{N} \text { - live, } 3250 \mathrm{~g}\end{array}$ & 6 & Cefoxitin (5d) \\
\hline 4 & 33 & 24.22 & $\mathrm{G} 2 \mathrm{PO}$ & Prematurity, PROM & $\begin{array}{l}\text { Caesarean } \\
\text { section: } \\
34+1 \text { weeks }\end{array}$ & $\begin{array}{l}\text { Combined } \\
\text { spinal epidural }\end{array}$ & $9-10-10$ & $\begin{array}{l}M \text { - good } \\
N \text { - live, } 2200 \mathrm{~g}\end{array}$ & 12 & $\begin{array}{l}\text { Cefmetazole } \\
(12 \mathrm{~d})\end{array}$ \\
\hline 5 & 24 & 23.37 & $\mathrm{G} 2 \mathrm{PO}$ & None & $\begin{array}{l}\text { Caesarean } \\
\text { section: } \\
39+2 \text { weeks }\end{array}$ & $\begin{array}{l}\text { Combined } \\
\text { spinal epidural }\end{array}$ & $10-10-10$ & $\begin{array}{l}M \text { - good } \\
\mathrm{N} \text { - live, } 3600 \mathrm{~g}\end{array}$ & 4 & Cefoxitin (2d) \\
\hline 6 & 21 & 22.60 & G1P0 & $\begin{array}{l}\text { Prematurity, } \\
\text { Moderate anemia }\end{array}$ & $\begin{array}{l}\text { Caesarean } \\
\text { section: } \\
33+6 \text { weeks }\end{array}$ & $\begin{array}{l}\text { General } \\
\text { anesthesia }\end{array}$ & $8-9-10$ & $\begin{array}{l}M \text { - good } \\
\mathrm{N} \text { - live, } 2180 \mathrm{~g}\end{array}$ & 10 & Cefoxitin (7d) \\
\hline 7 & 19 & 19.56 & G1P0 & Prematurity & $\begin{array}{l}\text { Caesarean } \\
\text { section: } \\
34 \text { weeks }\end{array}$ & $\begin{array}{l}\text { Combined } \\
\text { spinal epidural }\end{array}$ & $8-9-10$ & $\begin{array}{l}M \text { - good } \\
N \text { - live, } 2260 \mathrm{~g}\end{array}$ & 8 & $\begin{array}{l}\text { Piperacillin- } \\
\text { tazobactam (7d) }\end{array}$ \\
\hline 8 & 25 & 18.43 & G1P0 & None & $\begin{array}{l}\text { Terminate } \\
\text { pregnancy: } \\
17 \text { weeks }\end{array}$ & $\begin{array}{l}\text { Combined } \\
\text { spinal epidural }\end{array}$ & None & $\begin{array}{l}\text { M - Cardiac shock, } \\
\text { transferred to the } \\
\text { Department of } \\
\text { Cardiology } \\
\mathrm{N} \text { - died, } 80 \mathrm{~g}\end{array}$ & 3 & Ceftriaxone(2d) \\
\hline 9 & 19 & 20.30 & G1P0 & Prematurity & $\begin{array}{l}\text { Caesarean } \\
\text { section: } \\
30+4 \text { weeks }\end{array}$ & $\begin{array}{l}\text { General } \\
\text { anesthesia }\end{array}$ & $4-5-8$ & $\begin{array}{l}\mathrm{M} \text { - died with } \\
\text { pulmonary infection } \\
\text { and heart failure } \\
\mathrm{N} \text { - live, } 1095 \mathrm{~g} \text {, } \\
\mathrm{SGA} \text {, died in the } \\
\text { following day }\end{array}$ & 8 & $\begin{array}{l}\text { Cefoxitin }(2 d) \\
\text { Meropene }(5 d)\end{array}$ \\
\hline 10 & 29 & 25.20 & G2P0 & Prematurity, PROM & $\begin{array}{l}\text { Caesarean } \\
\text { section: } \\
36+5 \text { weeks }\end{array}$ & $\begin{array}{l}\text { Combined } \\
\text { spinal epidural }\end{array}$ & $10-10-10$ & $\begin{array}{l}M \text { - good } \\
N \text { - live,2930 g }\end{array}$ & 3 & Cefoxitin (2d) \\
\hline 11 & 30 & 23.44 & G5P1 & $\begin{array}{l}\text { Prematurity, MCDA, } \\
\text { Placenta praevia, } \\
\text { Postpartum } \\
\text { hemorrhage }\end{array}$ & $\begin{array}{l}\text { Caesarean } \\
\text { section: } \\
34+4 \text { weeks }\end{array}$ & $\begin{array}{l}\text { Combined } \\
\text { spinal epidural }\end{array}$ & N1: 9-10-10 & $\begin{array}{l}\text { M - good, } \\
\text { with pulmonary } \\
\text { infection } \\
\mathrm{N} \text { (twins) - 1: live, } \\
1550 \mathrm{~g} \text {, with SGA; } \\
\text { 2: died, } 2080 \mathrm{~g} \text {, } \\
\text { with TOF }\end{array}$ & 46 & $\begin{array}{l}\text { Meropene (14d) } \\
\text { Piperacillin } \\
\text { tazobactam (7d) }\end{array}$ \\
\hline 12 & 34 & 26.53 & G6P1 & $\begin{array}{l}\text { Breech } \\
\text { presentation }\end{array}$ & $\begin{array}{l}\text { Caesarean } \\
\text { section: } \\
38+5 \text { weeks }\end{array}$ & $\begin{array}{l}\text { Combined } \\
\text { spinal epidural }\end{array}$ & $10-10-10$ & $\begin{array}{l}M \text { - good } \\
\mathrm{N} \text { - live, } 2800 \mathrm{~g}\end{array}$ & 6 & Cefoxitin (2d) \\
\hline
\end{tabular}

VSD ventricular septal defect, PDA patent ductus arteriosus, $B M I$ body mass index $\left(\mathrm{kg} / \mathrm{cm}^{2}\right)$, PROM premature rupture of membranes, GDM gestational diabetes mellitus, MCDA monochorionic diamnionic twin, $M$ maternal, $N$ neonate, PFO patent foramen ovale, TOF Tetalogy of Fallot, HOD hospital day (total days in hospital)

Our subjects were asymptomatic before conception, or experienced mild symptoms, and physician did not pay enough attention to them. During pregnancy, the most common complications in our cases were pulmonary arterial hypertension, atrioventricular valve diseases and arrhythmia. No incidence of thromboembolism was found, and this may have assisted our clinical outcomes, which were basically good in spite of varied degrees of pulmonary arterial hypertension in 7 patients. The numbers of patients in the subgroups were too small to conduct further statistical analysis.

Asymptomatic patients with MVSA should be followed closely for potential cardiac complications. This is especially important for the cases in the setting of pregnancy [15-17]. Pregnancy places extra load on the heart, and can affect cardiac function, leading to increased maternal morbidity and perinatal morbidity $[18,19]$. In general, women with NYHA class > III have 
Table 3 Outcome of pregnancies with different maternal cardiac anomaly

\begin{tabular}{|c|c|c|c|c|c|c|}
\hline $\begin{array}{l}\text { Parameter } \\
\text { of anomaly }\end{array}$ & $\begin{array}{l}\text { Number.of case } \\
\text { (Fetal/maternal) }\end{array}$ & Prematurity & SGA & Fetal death & Neonatal death & Maternal death \\
\hline Total, n (\%) & $13 / 12$ & 7 (58.3\%) & $2(15.4 \%)$ & $1(7.7 \%)$ & $2(15.4 \%)$ & 1 (8.3\%) \\
\hline \multicolumn{7}{|l|}{ Size of VSA } \\
\hline$<10 \mathrm{~mm}$ & $2 / 2$ & $1(50 \%)$ & 0 & 0 & 0 & 0 \\
\hline $10-15$ mm & $5 / 5$ & $4(80 \%)$ & 0 & 0 & 0 & 0 \\
\hline$>15 \mathrm{~mm}$ & $6 / 5$ & $2(40 \%)$ & 2 (33.3\%) & $1(16.7 \%)$ & $2(33.3 \%)$ & $1(16.7 \%)$ \\
\hline \multicolumn{7}{|l|}{ Defect of VSA } \\
\hline Intact & $5 / 4$ & $1(25 \%)$ & $1(20 \%)$ & $1(20 \%)$ & $1(20 \%)$ & 0 \\
\hline$\leq 5 \mathrm{~mm}$ & $4 / 4$ & $3(75 \%)$ & 0 & 0 & 0 & 0 \\
\hline$>5 \mathrm{~mm}$ & $4 / 4$ & $3(75 \%)$ & $1(25 \%)$ & 0 & $1(25 \%)$ & $1(25 \%)$ \\
\hline \multicolumn{7}{|l|}{ LVSD } \\
\hline Normal & $12 / 11$ & $7(63.6 \%)$ & $2(16.7 \%)$ & 0 & $2(16.7 \%)$ & $1(8.3 \%)$ \\
\hline Dysfunction & $1 / 1$ & 0 & 0 & $1(100 \%)$ & 0 & 0 \\
\hline \multicolumn{7}{|l|}{ NYHA-FC } \\
\hline$|-| \mid$ & $3 / 3$ & $1(33.3 \%)$ & 0 & 0 & 0 & 0 \\
\hline III-IV & $10 / 9$ & $6(66.7 \%)$ & $2(20 \%)$ & $1(10 \%)$ & $2(20 \%)$ & $1(10 \%)$ \\
\hline \multicolumn{7}{|l|}{$\mathrm{PH}$} \\
\hline None & $6 / 5$ & $3(60 \%)$ & $1(16.7 \%)$ & 0 & $1(16.7 \%)$ & 0 \\
\hline Mild & $3 / 3$ & 1 (33.3\%) & 0 & $1(33.3 \%)$ & 0 & 0 \\
\hline Moderate & $3 / 3$ & $2(66.7 \%)$ & 0 & 0 & 0 & 0 \\
\hline Severe & $1 / 1$ & $1(100 \%)$ & $1(100 \%)$ & 0 & $1(100 \%)$ & $1(100 \%)$ \\
\hline \multicolumn{7}{|l|}{ Congenital abnormality } \\
\hline None & $5 / 4$ & $2(50 \%)$ & $1(20 \%)$ & $1(20 \%)$ & $1(20 \%)$ & 0 \\
\hline VSD & $7 / 7$ & $5(71.4 \%)$ & $1(14.3 \%)$ & 0 & $1(14.3 \%)$ & $1(14.3 \%)$ \\
\hline PDA & $1 / 1$ & 0 & 0 & 0 & 0 & 0 \\
\hline \multicolumn{7}{|l|}{ Valve condition } \\
\hline Normal & $4 / 4$ & $3(75 \%)$ & 0 & 0 & 0 & 0 \\
\hline TR & $4 / 4$ & $2(50 \%)$ & $1(25 \%)$ & 0 & $1(25 \%)$ & $1(25 \%)$ \\
\hline$M R$ & $1 / 1$ & $1(100 \%)$ & 0 & 0 & 0 & 0 \\
\hline $\mathrm{TR}+\mathrm{MR}$ & $4 / 3$ & $1(33.3 \%)$ & $1(25 \%)$ & $1(25 \%)$ & $1(25 \%)$ & 0 \\
\hline \multicolumn{7}{|l|}{ Arrhythmia } \\
\hline None I & $5 / 5$ & $3(60 \%)$ & 0 & 0 & 0 & 0 \\
\hline Sinus tachycardia & $4 / 4$ & $2(50 \%)$ & 0 & 0 & 0 & 0 \\
\hline$I^{\circ} A V B$ & $1 / 1$ & 0 & 0 & $1(100 \%)$ & 0 & 0 \\
\hline IRBBB & $1 / 1$ & $1(100 \%)$ & $1(100 \%)$ & 0 & $1(100 \%)$ & $1(100 \%)$ \\
\hline Sinus tachycardia + APB & $2 / 1$ & 1 (100\%) & $1(50 \%)$ & 0 & 1 (50\%) & 0 \\
\hline
\end{tabular}

NYHA-FC cardiac function grading (New York Heart Association), $P H$ pulmonary hypertension, VSD ventricular septal defect, PDA patent ductus arteriosus, TR tricuspid regurgitation, MR mitral regurgitation, AVB atrioventricular block, IRBBB incomplete right bundle branch block, SGA Small for gestational age

a relatively poor prognosis during pregnancy [20]. In our study, one case with NYHA class IV and severe pulmonary hypertension had to terminate pregnancy at 17 weeks of gestation; and one case with NYHA class IV expired.

We assumed that pregnant women with MVSA were at risk for maternal and neonatal complications, although the outcome in pregnancy with MVSA has not been reported in the literature. There was indeed a high rate (> 80\%) of obstetric events involving prematurity, PROM, preeclampsia, breech presentation, GDM and postpartum hemorrhage. Mothers with CHD also have a high incidence of fetal complications such as miscarriage, premature births, low birth weights, respiratory distress syndrome, and cardiac anomalies [21]. In this study, neonatal congenital heart disease (Tetralogy of Fallot) and SGA at birth (1550 g at $34+4$ weeks, 
survived) occurred in a twin gestation, the former expired and the latter survived. An additional singleton baby was born with SGA (1095 $g$ at $30+4$ weeks gestation) and expired on day 2 post birth.

Due to high risk of complications during vaginal delivery, a cesarean section delivery is usually recommended for woman with cardiac disease, since extra cardiac burden from prolonged laboring process may be avoided $[17,22]$. In the current study, except one terminated pregnancy in the second trimester, all of the remaining patients delivered by cesarean section under combined spinal epidural anesthesia, epidural anesthesia or general anesthesia. Except one maternal death from pulmonary infection and heart failure (also with severe pulmonary arterial hypertension, NYHA class IV), all other patients survived. Almost all of them suffered cardiac complications such as mild-to-moderate pulmonary arterial hypertension, atrioventricular valve diseases and arrhythmia, but none of them developed thromboembolism. The relative good outcomes in our patients may be at least partially owing to the absence of severe hypertension, cardiac failure and cerebral and pulmonary embolism. In addition, comprehensive interdisciplinary management among the cardiologist, obstetrician, anesthetist, and neonatologist, and detailed plans for delivery may have improved the prognosis [23].

\section{Conclusion}

Pregnancy with MVSA presents an increased risk of cardiac complications. Maternal and neonatal risks seem to be associated with heart functional classification, pulmonary hypertension and history of serious cardiac arrhythmia. Accurate diagnosis and care by a multidisciplinary team should be the best approach in order to improve prognosis in these patients.

\section{Abbreviations \\ AVB: Atrioventricular block; CHD: Congenital heart disease; GDM: Gestational diabetes mellitus; IRBBB: Incomplete right bundle branch block; MVSA: Membranous ventricular septal aneurysm; NYHA-FC: Cardiac function grading (New York Heart Association); PDA : Patent ductus arteriosus; PFO: Patent foramen ovale; PH: Pulmonary hypertension; PROM: Premature rupture of membranes; SGA: Small for gestational age; VPB: Ventricular premature beat; VSD: Ventricular septal defect}

\section{Acknowledgements}

We feel grateful for the doctors and staff who have been involved in this work. We also thank Dr. John J Xin (University of Chicago) for scientific discussion and editing assistance.

\section{Funding}

This work was supported by the Science Foundation of Sichuan Province

(2014JY0258) and the Science Foundation of Chengdu (2014-HM01-00039-SF).

\section{Availability of data and materials}

The datasets used and/or analysed during the current study available from the corresponding author on reasonable request.

\section{Authors' contributions}

KNW carried out the retrospective review of all cases, participated in data analysis and writing manuscript. HYY supervised the whole study, and carried out the study design and correction of the manuscript. XDW and XHL participated in the design of the study. AYX and YY participated the analysis of cases. All authors read and approved the final manuscript.

\section{Ethics approval and consent to participate}

This study was approved by the Institutional Review Board of West China Second University Hospital.

\section{Consent for publication}

Written informed consent was obtained from each patient or parent for publication of patient's individual identifiable data. A copy of the written consent is available for review by the Editor of this journal.

\section{Competing interests}

The authors declare that they have no competing interests.

\section{Publisher's Note}

Springer Nature remains neutral with regard to jurisdictional claims in published maps and institutional affiliations.

Received: 4 December 2016 Accepted: 21 December 2017 Published online: 03 January 2018

\section{References}

1. Mügge A, Daniel WG, Angermann C, Spes C, Khandheria BK, Kronzon I, et al. Atrial septal aneurysm in adult patients. A multicenter study using transthoracic and transesophageal echocardiography. Circulation. 1995; 91(11):2785-92.

2. Lazarev SM, Matsan VI. Aneurysm of the membranous heart septum. Kardiologia. 1989;29:36-40.

3. Razzouk L, Applebaum RM, Okamura C, Saric M. The windsock syndrome: subpulmonic obstruction by membranous ventricular septal aneurysm in congenitally corrected transposition of great arteries. Echocardiography. 2013:30(8):E243-8

4. Laennec RHT. Trait de L'auscultation Mediate et des Maladies des Poumons et du Coeur. 2nd ed. Paris: J.S. Chaude; 1826.

5. Choi M, Jung Jl, Lee BY, Kim HR. Ventricular septal aneurysms in adults: findings of cardiac CT images and correlation with clinical features. Acta Radiol. 2011:52(6):619-23.

6. Loukas M, Shane Tubbs R, Louis RG Jr, Curry B. Pseudoaneurysm of the membranous septum: case report and review of the literature. Surg Radiol Anat. 2006;28:564-8

7. Freedom RM, White RD, Pieroni DR, Varghese PJ, Krovetz LJ, Rowe RD. The natural history of the so-called aneurysm of the membranous ventricular septum in childhood. Circulation. 1974;49:375-84.

8. Yilmaz AT, Ozal E, Arslan M, Tatar H, Ozturk OY. Aneurysm of the membranous septum in adult patients with perimembranous ventricular septal defect. Eur J Cardiothorac Surg. 1997;11:307-11.

9. Love BA, Parness IA, Fuster V. Membranous septal aneurysm causing right ventricular outflow tract obstruction. Clin Cardiol. 2009;32(12):E87.

10. Salazar J, Gutierrez A, Cay E, Ballester C, Salazar JJ, Placer L. Cerebral embolism and thrombus in a membranous interventricular septal aneurysm. Ann Thorac Surg. 2003;76(1):286-7.

11. Horne D, White CW, Mackenzie GS, Kirkpatrick ID, Freed DH. Adult presentation with a bilobed membranous ventricular septal aneurysm. Can J Cardiol. 2013:29(7):893.e1-3.

12. Haegeli LM, Ercin E, Steffel J, Wolber T, Tanner FC, Jenni R, et al. Incidence and prognosis of ventricular arrhythmias in patients with congenital left ventricular aneurysms or diverticula. Am J Med. 2015;128(6):653.e1-6.

13. Naidu A, Ricketts M, Goela A, Shoemaker G, Li S. Incidental discovery of a membranous ventricular septal aneurysm in two dissimilar patients. Case Rep Cardiol. 2012;2012:324326.

14. Yavuz S, Eris C, Goncu T, Sezen M, Ata Y, Turk T. An incidental aneurysm of the interventricular membranous septum. Arch Iran Med. 2010;13(4):363-4.

15. Krexi D, Sheppard MN. Pulmonary hypertensive vascular changes in lungs of patients with sudden unexpected death. Emphasis on congenital heart disease, Eisenmenger syndrome, postoperative deaths and death during pregnancy and postpartum. J Clin Pathol. 2015;68(1):18-21. 
16. Stendahl JC, Hasan AS, Simegn MA. Massive interventricular septal aneurysm and stroke in a healthy young patient: guilt by association? J Stroke Cerebrovasc Dis. 2014;23(3):590-1.

17. Warnes CA. Pregnancy and delivery in women with congenital heart disease. Circ J. 2015;79(7):1416-21.

18. Lu CW, Shih JC, Chen SY, Chiu HH, Wang JK, Chen CA, et al. Comparison of 3 risk estimation methods for predicting cardiac outcomes in pregnant women with congenital heart disease. Circ J. 2015;79(7):1609-17.

19. Hink E, Bolte AC. Pregnancy outcomes in women with heart disease: experience of a tertiary center in the Netherlands. Pregnancy Hypertens. 2015:5(2):165-70.

20. Harris IS. Management of Pregnancy in patients with congenital heart disease. Prog Cardiovasc Dis. 2011;53(4):305-11.

21. Drenthen W, Pieper PG, Roos-Hesselink JW, van Lottum WA, Voors AA, Mulder BJ, et al. Outcome of pregnancy in women with congenital heart disease: a literature review. J Am Coll Cardiol. 2007;49(24):2303-11.

22. Wang $\mathrm{K}$, Luo H, Xin Y, Yu H. Successful pregnancy and delivery in patients with uncorrected single ventricle: three new cases and literature review. Int J Cardiol. 2015;184:135-9.

23. Harris RC, Fries MH, Boyle A, Adeniji-Adele H, Cherian Z, Klein N, et al. Multidisciplinary management of pregnancy in complex congenital heart disease: a model for coordination of care. Congenit Heart Dis. 2014;9(6): E204-11.

\section{Submit your next manuscript to BioMed Central} and we will help you at every step:

- We accept pre-submission inquiries

- Our selector tool helps you to find the most relevant journal

- We provide round the clock customer support

- Convenient online submission

- Thorough peer review

- Inclusion in PubMed and all major indexing services

- Maximum visibility for your research

Submit your manuscript at www.biomedcentral.com/submit 